\title{
Spontaneous disinsertion of an iris-fixated 3-piece intraocular lens haptic from the optic in a patient with pathologic myopia
}

\author{
Daniel P Greene ${ }^{1}$ and Seth W Meskin ${ }^{2,3}$ \\ ${ }^{1}$ Richmond Eye Associates, Glen Allen, Virginia USA \\ ${ }^{2}$ Department of Ophthalmology and Visual Science, Yale University School of Medicine, New Haven, Connecticut USA \\ ${ }^{3}$ Eye Physicians \& Surgeons, Milford, Connecticut USA
}

\begin{abstract}
A 39-year-old man with pathologic myopia and a dislocated native lens of the right eye underwent pars plana lensectomy and implantation of an iris-fixated 3-piece MA60MA foldable acrylic intraocular lens with -5.0 diopter power. The patient presented 11 months later with spontaneous haptic disinsertion from the optic. The meniscus (concave-convex) configuration of this iris-fixated lens may have contributed to spontaneous haptic-optic disjunction. This is the first such case reported involving a negative diopter power intraocular lens.
\end{abstract}

\section{Introduction}

Dislocation of an intraocular lens (IOL) haptic from the optic has been reported to occur during surgery, after trauma, and spontaneously $[1,2]$. Spontaneous dislocations are rare, with only 8 cases reported in the literature [3-8]. Such cases can occur acutely or many years following cataract surgery, and have been described in lenses placed in the capsular bag, sulcus, or posterior to the iris with fixation sutures. None of the previously reported cases have involved a negative diopter power lens.

We describe a case of spontaneous disinsertion of a haptic from a 3 -piece iris-fixated IOL in a patient with pathologic myopia. To our knowledge, this is the second such case involving an iris-fixated lens, and the first case implicating a lens with negative dioptric power.

\section{Case report}

A 39-year-old man with a history of pathologic myopia, amblyopia, and trauma of the right eye was referred for a posteriorly dislocated crystalline lens. Medical history was significant for hypertension, hypercholesterolemia, and insulin-dependent diabetes mellitus. On exam, the patient had a proptotic right globe due to axial myopia. Motility testing revealed an intermittent exotropia of the right eye. Visual acuity was counting fingers in the right eye (improving to $20 / 300$ on potential acuity meter testing) and 20/30 in the left eye. Intraocular pressure (IOP) was $18 \mathrm{~mm} \mathrm{Hg}$ in both eyes. The right eye demonstrated a completely dislocated crystalline lens into the vitreous cavity, iridodonesis, and vitreous in the anterior chamber. The cornea was clear. Fundus exam revealed mild non-proliferative diabetic retinopathy in both eyes. No posterior staphyloma was noted. Axial length was $33.45 \mathrm{~mm}$ and $23.21 \mathrm{~mm}$ in the right and left eyes, respectively.

Various treatment options were discussed including observation due to limited visual prognosis, contact lens use, or pars planavitrectomy and lensectomy with possible concurrent secondary IOL insertion. The patient was advised to address the dislocated lens to prevent retinal or ocular complications with recommendation to leave the eye aphakic and use a contact lens postoperatively. The patient adamantly refused use of contact lens and requested surgical implantation of an IOL. Selection of an IOL was limited by the need for a negative powered lens calculated by biometry.

The patient underwent uncomplicated combined pars plana vitrectomy, pars plana lensectomy, and implantation of an iris-fixated 3-piece acrylic posterior chamber IOL (-5.0 diopter, MA60MA, Alcon Surgical). He did well in the early post-operative period other than requiring medical management of temporary IOP spike. The IOL was stable and centered.

The patient was then lost to follow-up until presenting 11 months later with worsening vision in the right eye. Examination noted complete disinsertion of the superior haptic from the optic junction with the superior haptic still secured to the iris via prolene suture (Figure 1). The remaining optic and inferior haptic were dislocated into the vitreous cavity. The intact inferior prolene suture was still visible in the iris. The patient denied any recent trauma. IOL removal was recommended at that time, but the patient was again lost to followup.

Correspondence to: Daniel P. Greene, MD, Richmond Eye Associates, 4600 Cox Road, Suite 120, Glen Allen, VA 23060, USA, Tel: 804-270-0330; E-mail: dpgreene@gmail.com

Keywords: haptic-optic disinsertion, intraocular lens, myopia

Received: October 12, 2015; Accepted: October 26, 2015; Published: October 30, 2015 


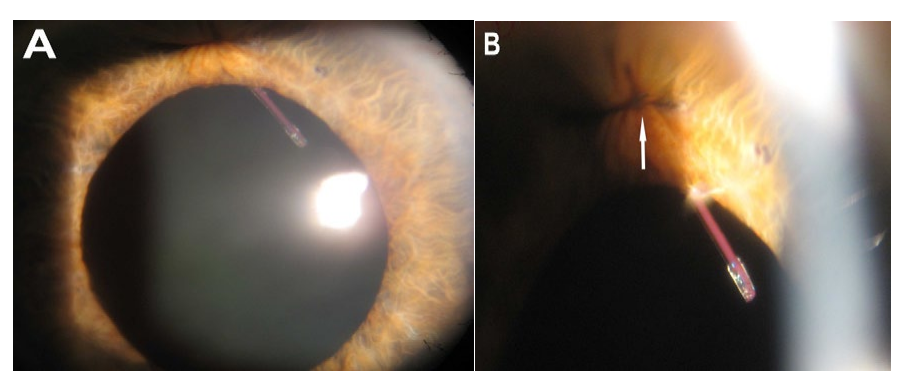

Figure 1. A. Slit lamp photograph of the right eye centered on the pupil shows a 3-piece intraocular lens haptic with no visible optic. B. Magnified view centered on the superior iris shows the same haptic with an intact iris prolene suture (arrow).

\section{Discussion}

Spontaneous haptic disinsertion from an IOL occurs rarely, but has previously involved various types of lens models found at different implantation sites [1-8]. Our case involved a foldable acrylic 3-piece IOL that was fixated to the iris since the capsular bag and native lens had become completed dislocated, presumably as a result of his high axial myopia or from possible unreported trauma. Known complications of 3-piece iris-fixated lenses include haptic slippage from the suture and spontaneous haptic-optic disjunction $[4,9]$.

Our patient's cataract surgery was complicated by his history of unilateral pathologic myopia with high axial length $(33.45 \mathrm{~mm})$, which may have contributed to haptic-optic disjunction. The -5.0 diopter lens power chosen for implantation has a "meniscus" (convex-concave) lens shape in order to produce negative power [10]. It is possible that the manufacturing process or optic configuration required for these lenses could produce haptic-optic junctions that are less durable than those found in biconvex lenses. In response to our inquiry regarding this case, Alcon Medical Information Services informed us that other cases of spontaneous dislocation of the optic-haptic junction involving the MA60MA lens have been reported and stated that special consideration should be given to dimensions of lenses at the extreme ends of the power range [11].

Although not reported by the patient, we cannot exclude the possibility of trauma contributing to haptic disinsertion. Additionally, the patient's iridodonesis in the setting of a highly myopic, vitrectomized eye might have contributed to lens movement and optic/ haptic junction failure. We believe, however, that haptic slippage from the suture would have been more likely to occur rather than disjunction if this case was due to iridodonesis alone. Our report is limited by the patient's lack of follow-up care, which precluded the surgeon from removing the lens to analyze for possible manufacturing error.
Reports in the literature of complications after implantation of a negative powered IOL are rare. Based on the findings in this case, cataract surgeons may consider alternative strategies for secondary IOL placement in patients with severe myopia requiring a lens with a negative power.

\section{Authorship and contribution}

All authors listed on the title page made significant contributions to this manuscript.

\section{Acknowledgements}

None

\section{Funding}

No funding was received for this study.

\section{Competing interest}

The authors have no conflict of interest or financial relationships related to this manuscript.

\section{References}

1. Das S, Patil MD, Garg P (2007) Traumatic fracture of posterior chamber intraocular lens. J Cataract Refract Surg 33:2151-2152. [Crossref]

2. Eleftheriadis H, Sahu D, Willekens B, Liu CSC (2001) Corneal decompensation and graft failure secondary to a broken posterior chamber poly(methyl methacrylate) intraocular lens haptic. J Cataract Refract Surg27:2047-2050. [Crossref]

3. Chuang C, Lee J, Lin K, Hsiao C (2010) Late-onset spontaneous haptic disinsertion from a three-piece intraocular lens. Jpn J Ophthalmol 54: 499-516.

4. Condon GP, Masket S, Kranemann C, Crandall AS, Ahmed II (2007) Small-incision iris fixation of foldable intraocular lenses in the absence of capsule support. Ophthalmology 114:1311-1318. [Crossref]

5. Fujishima K, Yoshitomi F, Oshika T (2002) Spontaenous disinsertion of ahpatic from a 3-piece acrylic foldable intraocular lens after surgery. J Cataract Refract Surg 28: 1296-1298. [Crossref]

6. Por YM, Chee S (2004) Spontaneous disinsertion of a multipieceflodable acrylic intraocular lens haptic 3 and 12 months after implantation. J Cataract Refract Surg 30: 1139-1142. [Crossref]

7. Sandhu R, Hunter P (2005) Late spontaneous prolapse of a posterior chamber intraocular lens. J Cataract Refract Surg 31: 2425-2426. [Crossref]

8. Solano JM, Baratz KH, Mahr MA, Erie JC (2007) Late spontaneous haptic disinsertion from a three-piece intraocular lens. Am J Ophthalmol 143: 521-522. [Crossref]

9. Kaiura TL, Seedor JA, Koplin RS, Rhee MK, Ritterband DC (2005) Complications arising from iris-fixated posterior chamber intraocular lenses. J Cataract Refract Surg 31: $2420-2422$. [Crossref]

10. Holladay JT (1986) Evaluating the intraocular lens optic. Surv Ophthalmol 30: 385390

11. Alcon Medical Information Services, personal communication. August 6, 2013

Copyright: $\odot 2015$ Greene DP. This is an open-access article distributed under the terms of the Creative Commons Attribution License, which permits unrestricted use, distribution, and reproduction in any medium, provided the original author and source are credited. 